TRANSACTIONS OF THE

AMERICAN MATHEMATICAL SOCIETY

Volume 315, Number 1, September 1989

\title{
HAMBURGER MOMENT PROBLEMS AND ORTHOGONAL POLYNOMIALS
}

\author{
T. S. CHIHARA
}

\begin{abstract}
We consider a sequence of orthogonal polynomials given by the classical three term recurrence relation. We address the problem of deciding the determinacy or indeterminacy of the associated Hamburger moment problem on the basis of the behavior of the coefficients in the three term recurrence relation. Comparisons are made with other criteria in the literature. The efficacy of the criteria obtained is illustrated by application to many specific examples of orthogonal polynomials.
\end{abstract}

\section{INTRODUCTION}

The coefficients in the classical three term recurrence formula satisfied by a sequence of orthogonal polynomials contain a great deal of information about the spectral properties of the polynomials. In particular, information concerning the determinacy-indeterminacy of the moment problems associated with the orthogonal polynomials can sometimes be decided on the basis of the behavior of these coefficients. In this paper, we wish to add to the limited store of criteria that permit such deductions.

We consider the orthogonal polynomial sequence $\left\{P_{n}(x)\right\}$ given by the usual three term recurrence relation

$$
\begin{aligned}
& P_{n}(x)=\left(x-c_{n}\right) P_{n-1}(x)-\lambda_{n} P_{n-2}(x), \\
& P_{-1}(x)=0, \quad P_{0}(x)=1, \quad c_{n} \text { real, } \lambda_{n}>0, n \geq 1 .
\end{aligned}
$$

The recurrence $(1.1)$ determines the moments $\mu_{n}$ of a linear functional $\mathscr{L}$ over the algebra of polynomials:

$$
\mathscr{L}\left[x^{n}\right]=\mu_{n}, \quad n=0,1,2, \ldots .
$$

The $\mu_{n}$ are defined recursively by the conditions,

$$
\mathscr{L}[1]=\mu_{0}=\lambda_{1}, \quad \mathscr{L}\left[P_{n}(x)\right]=0, \quad n=1,2,3, \ldots
$$

Received by the editors March 10, 1988. Presented to the Society in San Antonio, Texas, on January 23, 1987.

1980 Mathematics Subject Classification (1985 Revision). Primary 42C05; Secondary 30E05, $33 \mathrm{~A} 65$.

Key words and phrases. Moment problems, orthogonal polynomials, recurrence relations, chain sequences.

Research supported by National Science Foundation grant DMS 8400701. 
The reality of the $c_{n}$ and the positivity of the $\lambda_{n}$ then ensures that Hamburger's condition is satisfied so there exists a solution $\psi$ of the resulting Hamburger moment problem:

$$
\int_{-\infty}^{\infty} x^{n} d \psi(x)=\mu_{n} .
$$

The polynomials defined by (1.1) are then orthogonal with respect to $\psi$ :

$$
\int_{-\infty}^{\infty} P_{m}(x) P_{n}(x) d \psi(x)=\lambda_{1} \lambda_{2} \cdots \lambda_{n+1} \delta_{m n} .
$$

The corresponding orthonormal polynomials are

$$
p_{n}(x)=\left(\lambda_{1} \lambda_{2} \cdots \lambda_{n+1}\right)^{-1 / 2} P_{n}(x), \quad n \geq 0 .
$$

We will assume that the distribution function $\psi$ has been normalized to have total mass $1\left(\lambda_{1}=1\right)$.

We now wish to address the question of the determinacy of the resulting Hamburger moment problem. Specifically, we wish to obtain criteria expressed in terms of the coefficients in (1.1) for deciding the determinacy or indeterminacy of the Hamburger moment problem. Probably the best known criterion of this type is due to Carleman [4; 14]. Carleman's theorem says that the moment problem is determined if

$$
\sum \lambda_{n}^{-1 / 2}=\infty
$$

Our approach is based upon use of chain sequences and as such limits our results to cases where the spectrum is restricted to a half-line. Thus our results are more limited than those of Carleman and Dennis and Wall [12] (see also §5) which predict determinacy of the Hamburger moment problem without assuming any bounds on the spectrum. On the other hand, our theorems are applicable to many cases for which the aforementioned criteria give no information. Additionally, our methods also yield criteria that predict indeterminacy.

\section{Preliminaries, AND notation}

We will establish the basic relations and background facts we will use. In particular, we will rederive certain results obtained earlier in [6] stating them in a somewhat more general and more easily applied form. This will also afford us the opportunity to correct some annoying misprints that appeared in [6]. We will use the notation

$$
\alpha_{n}(x)=\frac{\lambda_{n+1}}{\left(c_{n}-x\right)\left(c_{n+1}-x\right)}, \quad \alpha_{n}=\alpha_{n}(0) .
$$

A major role played by the sequence $\left\{\alpha_{n}(x)\right\}_{n=1}^{\infty}$ is explained by the following fundamental result $[5 ; 8]$ : 
Theorem 1. The true interval of orthogonality of the polynomials defined by (1.1) is a subset of $[c, \infty)$ if and only if (i) $c_{n}>c(n \geq 1)$ and (ii) $\left\{\alpha_{n}(c)\right\}$ is a chain sequence.

We recall that a sequence $\left\{a_{n}\right\}_{n=1}^{\infty}$ is a chain sequence if there exists a second sequence $\left\{g_{n}\right\}_{n=0}^{\infty}$ such that

$$
\begin{gathered}
0 \leq g_{0}<1, \quad 0<g_{n}<1, \quad n \geq 1 ; \\
a_{n}=\left(1-g_{n-1}\right) g_{n}, \quad n \geq 1 .
\end{gathered}
$$

The $g_{n}$ are called the parameters of the chain sequence. If $g_{0}=0$, the resulting parameters are the minimal parameters of the chain sequence and the resulting sequence is dominated by every other parameter sequence [8]. Except in $\S 5$ when we discuss the symmetric case, we will restrict ourselves to the cases where

(i) $\lim _{n \rightarrow \infty} c_{n}=\infty$;

(ii) there exists a real $c$ such that $\left\{\alpha_{n}(c)\right\}$ is a chain sequence.

We mention that in [7] we observed that if (2.2)(ii) holds, then (after omitting any $c_{n}$ that vanish) the divergence of $\sum c_{n}^{-1}$ is sufficient to conclude that the Hamburger moment problem is determined. Thus we will gain no further generality by replacing (2.2)(i) by $\lim \sup _{n \rightarrow \infty} c_{n}=\infty$.

A sufficient condition for (2.2)(ii) is that $\left\{\alpha_{n}\right\}$ is an "eventual chain sequence." That is, it is sufficient that there is an index $N$ such that $\left\{\alpha_{N+n}\right\}_{n=1}^{\infty}$ is a chain sequence. In turn, a sufficient condition for the latter is $[9, \mathrm{p} .362]$

$$
\alpha_{n} \leq \frac{1}{4}+\frac{1}{16 n^{2}}+O\left(n^{-3}\right) \text {. }
$$

On the other hand, a necessary condition for $(2.2)$ is $\liminf _{n \rightarrow \infty} \alpha_{n} \leq \frac{1}{4}[5 ; 8]$. Let $x_{n 1}<x_{n 2}<\cdots<x_{n n}$ denote the zeros of $P_{n}(x)$ and set

$$
\xi_{i}=\lim _{n \rightarrow \infty} x_{n i}, \quad \eta_{1}=\lim _{n \rightarrow \infty} x_{n n}, \quad \sigma=\lim _{i \rightarrow \infty} \xi_{i} .
$$

When the Hamburger moment problem is determined, $\sigma$ is the least limit point in the extended real number system of the spectrum of $\psi$.

Assume conditions (2.2) so that

$$
\xi_{1}>-\infty, \quad \eta_{1}=\infty
$$

Pick any fixed $c \leq \xi_{1}$ and let $m_{n}=m_{n}(c)$ denote the minimal parameters for the chain sequence $\left\{\alpha_{n}(c)\right\}$ :

$$
\alpha_{n}(c)=\left(1-m_{n-1}\right) m_{n}, \quad m_{0}=0,0<m_{n}<1(n \geq 1) .
$$

The zeros of $P_{n}(x)$ lie in $\left[\xi_{1}, \infty\right) \subseteq[c, \infty)$ so we set

$$
Q_{n}(x)=P_{n}(x-c) \text {. }
$$

Then $\left\{Q_{n}(x)\right\}$ satisfies (1.1) with $c_{n}$ replaced by

$$
c_{n}^{*}=c_{n}-c \text {. }
$$


Since the zeros of $Q_{n}(x)$ lie in $[0, \infty)$, there is a corresponding sequence of symmetric orthogonal polynomials $\left\{S_{n}(x)\right\}$ such that

$$
S_{2 n}(x)=Q_{n}\left(x^{2}\right) \text {. }
$$

Also, $S_{2 n+1}(x)=x K_{n}\left(x^{2}\right)$ where $\left\{K_{n}(x)\right\}$ is the sequence of monic kernel polynomials corresponding to $\left\{Q_{n}(x)\right\}[8$, p. 41].

These symmetric orthogonal polynomials satisfy the recurrence [8, pp. 45-46]

$$
S_{n}(x)=x S_{n-1}(x)-\gamma_{n} S_{n-2}(x),
$$

where $c_{1}^{*}=\gamma_{2}$ and

$$
c_{n+1}^{*}=\gamma_{2 n+1}+\gamma_{2 n+2}, \quad \lambda_{n+1}^{\cdot}=\gamma_{2 n} \gamma_{2 n+1}, \quad n \geq 1 \text {. }
$$

The $c_{n}^{*}$ and the $\gamma_{n}$ are further related through the parameters of the chain sequence in (2.1):

$$
\gamma_{2 n-1}=m_{n-1} c_{n}^{*}, \quad \gamma_{2 n}=\left(1-m_{n-1}\right) c_{n}^{*}, \quad n \geq 1 .
$$

We have

$$
Q_{n}(0)=S_{2 n}(0)=(-1)^{n} \gamma_{2} \cdots \gamma_{2 n}
$$

so for the corresponding orthonormal polynomials, $q_{n}(x)$, we have (cf. (1.10))

$$
\left[q_{n}(0)\right]^{2}=\frac{\gamma_{2} \cdots \gamma_{2 n}}{\gamma_{3} \cdots \gamma_{2 n+1}}=\frac{\left(1-m_{1}\right) \cdots\left(1-m_{n-1}\right) c_{1}^{*}}{m_{1} m_{2} \cdots m_{n} c_{n+1}^{*}} .
$$

Next we introduce the "numerator polynomials" $Q_{n}^{(1)}(x)$ :

$$
\begin{aligned}
& Q_{n}^{(1)}(x)=\left(x-c_{n+1}^{*}\right) Q_{n-1}^{(1)}(x)-\lambda_{n+1} Q_{n-2}^{(1)}(x), \\
& Q_{-1}^{(1)}(x)=0, \quad Q_{0}^{(1)}(x)=1 .
\end{aligned}
$$

Now it is clear that $\left\{\alpha_{n}^{(1)}(c)\right\}_{n=1}^{\infty}=\left\{\alpha_{n+1}(c)\right\}_{n=1}^{\infty}$ is a chain sequence for which the numbers $m_{n}^{(1)}=m_{n+1} \quad(n \geq 0)$ are nonminimal parameters (since $\left.m_{1}>0\right)$. Therefore, let $m_{n}^{*}=m_{n}^{*}(c)$ denote the corresponding minimal parameters:

$$
\alpha_{n}^{(1)}(c)=\left(1-m_{n-1}^{*}\right) m_{n}^{*}, \quad m_{0}^{*}=0,0<m_{n}^{*}<1, n \geq 1 .
$$

Note also that $m_{n}^{*}<m_{n+1}(n \geq 0)$ so that $\left\{m_{n}^{*}\right\}$ is never the maximal parameter sequence (that is, $\left\{\alpha_{n}^{(1)}(c)\right\}$ never determines its parameters uniquely).

Assuming the distribution for $\left\{Q_{n}^{(1)}(x)\right\}$ has also been normalized to have total mass 1 , we have corresponding to $(2.14)$

$$
\left[q_{n}^{(1)}(0)\right]^{2}=\frac{\left(1-m_{1}^{*}\right) \cdots\left(1-m_{n-1}^{*}\right) c_{2}^{*}}{m_{1}^{*} m_{2}^{*} \cdots m_{n}^{*} c_{n+2}^{*}} .
$$

Since $0<m_{n}^{*}<m_{n+1}(n \geq 1)$, it follows that

$$
\left[q_{n}^{(1)}(0)\right]^{2}>\frac{c_{2}^{*}}{c_{1}^{*}} \frac{m_{1}}{1-m_{1}}\left[q_{n+1}(0)\right]^{2} \text {. }
$$


Now, in general, a necessary and sufficient condition for the Hamburger moment problem associated with $\left\{Q_{n}(x)\right\}$ to be determined is the divergence of at least one of the two series

$$
\sum\left[q_{n}(0)\right]^{2}, \quad \sum\left[q_{n}^{(1)}(0)\right]^{2}
$$

$[14$, p. 68]. Since determinacy of a Hamburger moment problem is clearly invariant under translation, we conclude from (2.17) and (2.18) the following slightly more general restatement of our earlier theorem [6, Theorem 2].

Theorem 1. Let the coefficients in (1.1) satisfy the conditions (2.2). Let $\left\{m_{n}^{*}\right\}$ denote the minimal parameter sequence of the corresponding chain sequence (2.16). Then the Hamburger moment problem associated with (1.1) is determined if and only if

$$
\sum \frac{\left(1-m_{1}^{*}\right)\left(1-m_{2}^{*}\right) \cdots\left(1-m_{n-1}^{*}\right)}{m_{1}^{*} m_{2}^{*} \cdots m_{n}^{*}\left(c_{n+2}-c\right)}=\infty .
$$

\section{THE LIMIT CASE}

Maintaining the blanket hypotheses (2.2), we now specialize the coefficients in (1.1) to the case

$$
\lim _{n \rightarrow \infty} c_{n}=\infty, \quad \lim _{n \rightarrow \infty} \frac{\lambda_{n+1}}{c_{n} c_{n+1}}=L .
$$

Now if $L>\frac{1}{4},\left\{\alpha_{n}(c)\right\}$ is not a chain sequence for any $c$ and we would have $\sigma=-\infty\left[8\right.$, p. 109]. Thus we must have $L \leq \frac{1}{4}$. If $L<\frac{1}{4}$, then $\left\{\alpha_{n}\right\}$ is an eventual chain sequence and (2.2) is automatically satisfied. However, if $L=\frac{1}{4}$, then each of the three extremes, $\sigma=-\infty,|\sigma|<\infty, \sigma=\infty$, is possible [11] so we will have to impose the additional hypothesis (2.2)(ii) explicitly.

We continue to use the notation of $\S 2$, so that $\left\{m_{n}^{*}\right\}_{n=0}^{\infty}$ denotes the minimal parameter sequence of the chain sequence $\left\{\alpha_{n}^{(1)}(c)\right\}$. Now the parameters of a convergent chain sequence are themselves convergent [5; 8, pp. 102-103] so from (3.1), we conclude

$$
\begin{gathered}
\lim _{n \rightarrow \infty} m_{n}^{*}=\mu, \\
\mu=\frac{1}{2}[1-\sqrt{1-4 L}] .
\end{gathered}
$$

(The choice of the minus sign before the radical in (3.3) is justified by the observation that the $m_{n}^{*}$ are never maximal parameters.)

We write $P=\left\{P_{n}(x)\right\}$ and let $A_{n}=A_{n}(P)$ :

$$
A_{n}=\frac{\left(1-m_{1}^{*}\right) \cdots\left(1-m_{n-1}^{*}\right)}{m_{1}^{*} \cdots m_{n-1}^{*} m_{n}^{*}\left(c_{n+2}-c\right)}, \quad n>1 .
$$

We see that

$$
A_{n}^{1 / n} \begin{cases}\sim \frac{1-\mu}{\mu} c_{n}^{-1 / n}, & L \neq 0, \\ =\left[o\left(c_{n}^{1 / n}\right)\right], & L=0 .\end{cases}
$$


Combining the standard root test with Theorem 1, we conclude the following slightly more general restatement of our earlier result [6].

Theorem 2. Let

$$
\lim _{n \rightarrow \infty} c_{n}=\infty, \quad \lim _{n \rightarrow \infty} \frac{\lambda_{n+1}}{c_{n} c_{n+1}}=L \leq \frac{1}{4} .
$$

In case $L=\frac{1}{4}$, assume additionally that condition (2.2)(ii) is satisfied. The Hamburger moment problem associated with (1.1) is determined if

$$
\liminf _{n \rightarrow \infty} c_{n}^{1 / n}<\frac{1-\mu}{\mu}
$$

and it is indeterminate if

$$
\liminf _{n \rightarrow \infty} c_{n}^{1 / n}>\frac{1-\mu}{\mu} .
$$

Specializing further, we obtain the following convenient criteria:

Corollary. Let

$$
\lim _{n \rightarrow \infty} \frac{\lambda_{n+1}}{c_{n} c_{n+1}}=L<\frac{1}{4} .
$$

(i) If $c_{n}=O\left(n^{p}\right)$, the Hamburger moment problem is determined.

(ii) If $c_{n}=f_{n} q^{-n}$ where $0<q<1$ and $\left\{f_{n}\right\}$ is both bounded and bounded away from 0 , then the Hamburger moment problem is determined if $L<$ $q(q+1)^{-2}$ and is indeterminate if the opposite (strict) inequality holds.

Proof. (i) is immediate since $(1-\mu) / \mu>1$,

(ii) follows upon using (3.3) in (3.6) and (3.7).

The above are sufficiently general to decide immediately the determinacy or indeterminacy of many well-known examples. For example, for the six cases of Stieltjes-Carlitz polynomials that are orthogonal over $[0, \infty)[8$, pp. 193-195], we have in each case:

$$
c_{n}=4\left(k^{2}+1\right) \dot{n}^{2}+O(n), \quad \lambda_{n+1}=16 k^{2} n^{4}+O\left(n^{3}\right), \quad 0<k^{2}<1 .
$$

Thus $L=k^{2}\left(1+k^{2}\right)^{-2}<1 / 4$ so the determinacy of the Hamburger moment problems follow immediately.

For a second example, consider the generalized Stieltjes-Wigert polynomials $[8$, p. 174]. We have

$$
\begin{aligned}
& c_{n}=(1+q) q^{-3 / 2} q^{-2 n}+O\left(q^{-n}\right), \quad \lambda_{n+1}=\left(1-q^{n}\right)\left(1-p q^{n-1}\right) q^{-4 n}, \\
& p>0,0<q<1 .
\end{aligned}
$$

Thus, $L=q(1+q)^{-2}$ and the corollary gives the indeterminacy of the Hmp.

Finally, consider the Al-Salam and Carlitz polynomials [2; 8, p. 196]. This time we have

$$
\begin{aligned}
& c_{n+1}=(1+a) q^{-n}, \quad \lambda_{n+1}=a\left(1-q^{n}\right) q^{1-2 n}, \quad 0<q<1, a>0, \\
& \alpha_{n}=\frac{a}{(1+a)^{2}}\left(1-q^{n}\right) .
\end{aligned}
$$


Thus $\left\{\alpha_{n}\right\}$ increases monotonically to $L=a(1+a)^{-2} \leq 1 / 4$ so we conclude:

(i) the Hmp is determined if $a<q$ or $a>q^{-1}$;

(ii) the Hmp is indeterminate if $q<a<q^{-1}$.

The above leaves open the two cases: $a=q$ and $a=q^{-1}$. In both of these cases,

$$
L=\frac{q}{(1+q)^{2}} \quad \text { and } \quad \lim _{n \rightarrow \infty} c_{n}^{1 / n}=\frac{1}{q}=\frac{1-\mu}{\mu}
$$

To settle these cases, we will take advantage of the fact that the sequence $\left\{\alpha_{n}\right\}$ is increasing. We adopt the common convention that a prime on a summation symbol $\left(\sum^{\prime}\right)$ means that any terms with 0 in the denominator are omitted.

Theorem 3. Let $\alpha_{n}(c) \leq \alpha_{n+1}(c) \leq \frac{1}{4}, n \geq 2$. Then the Hmp is determined if

$$
\sum^{\prime}\left(\frac{1-\mu}{\mu}\right)^{n} \frac{1}{c_{n}}=\infty .
$$

Proof. The hypothesis assures us that $\left\{\alpha_{n}^{(1)}(c)\right\}$ is a nondecreasing chain sequence and this means that its minimal parameter sequence $\left\{m_{n}^{*}\right\}$ increases to the limit $\mu\left[8\right.$, p. 96]. Thus, $\left(1-m_{n}^{*}\right) / m_{n}^{*}>(1-\mu) / \mu$. Referring once again to $(3.4)$, we have

$$
A_{n}>\frac{1}{1-\mu}\left(\frac{1-\mu}{\mu}\right)^{n} \frac{1}{c_{n+2}},
$$

so another reference to Theorem 1 now completes the proof.

Returning to the Al-Salam and Carlitz polynomials, we take up the remaining cases, $a=q$ and $a=q^{-1}$. As we noted, in both cases, we have that $\left\{\alpha_{n}\right\}$ increases to $L=q(1+q)^{2}$ and $(1-\mu) / \mu=q^{-1}$. Since $c_{n}=O\left(q^{-n}\right)$, we conclude that the corresponding Hamburger moment problems are determined.

Remark. If in Theorem 3, we also assume that $L=1 / 4$, we conclude that the moment problem is determined if $\sum^{\prime} c_{n}^{-1}=\infty$. However, as we pointed out following (2.2), if $\left\{\alpha_{n}(c)\right\}$ is a chain sequence, the latter condition is sufficient for determinacy without further hypothesis.

Looking again at the Stieltjes-Carlitz polynomials, we consider the particular case, $c_{n+1}=(2 n+1)^{2}\left(k^{2}+1\right), \lambda_{n+1}=4\left(4 n^{2}-1\right) n^{2} k^{2}$. We have previously noted that the case $k^{2}>1$ can be reduced to the case $k^{2}<1$ whereas the limiting case $k^{2} \rightarrow 1$ leads to a different class of orthogonal polynomials [11, p. 638]. In fact, this limiting case turns out to be a special limiting case of Wilson's ${ }_{4} F_{3}$ orthogonal polynomials and Wilson has given their orthogonality relations [17]. Specifically, in this case we have $c_{n+1}=2(2 n+1)^{2}, \lambda_{n+1}=4 n^{2}\left(4 n^{2}-1\right)$ so that $\alpha_{n}=n^{2}\left(4 n^{2}-1\right)^{-1}>1 / 4$. The determinacy of the associated Hamburger moment problems cannot be determined by the theorems thus far obtained 
so we next obtain some results that will be applicable when $\alpha_{n} \geq 1 / 4$ and $\lim _{n \rightarrow \infty} c_{n}^{1 / n}=1$.

\section{AdDitionAl CRITERIA}

If $\alpha_{n}(c) \rightarrow L=\frac{1}{4} \quad\left(\mu=\frac{1}{2}\right)$, Theorem 2 only gives the indeterminacy criterion, $\liminf _{n \rightarrow \infty} c_{n}^{1 / n}>1$. On the other hand, if $L=0 \quad(\mu=0)$, Theorem 2 only provides the determinacy criterion, $\liminf _{n \rightarrow \infty} c_{n}^{1 / n}<\infty$. We therefore obtain here a few simple criteria that can be used to predict determinacy when $L=\frac{1}{4}$ and indeterminacy when $L=0$. We continue to maintain the notation of the previous sections. We also continue the blanket hypothesis that $(2.2)$ (ii) is satisfied. However, we mention that if $\alpha_{n}^{(1)}(c) \leq \beta_{n}$, where $\left\{\beta_{n}\right\}$ is a chain sequence, then it is always possible to choose $c^{\prime} \leq c$ so that $\left\{\alpha_{n}\left(c^{\prime}\right)\right\}$ is a chain sequence. Therefore, in (4.1) below, (2.2)(ii) need not be explicitly assumed as a separate hypothesis.

The new criteria are the result of application of the following basic result concerning chain sequences (see [8, pp. 95, 97]).

Lemma. Let $b=\left\{b_{n}\right\}$ be a chain sequence and let $0<a_{n} \leq b_{n}(n \geq 1)$. Then $\left\{a_{n}\right\}$ is also a chain sequence. If $m_{n}$ and $M_{n}$ are its minimal and maximal parameters, and if $\left\{h_{n}\right\}$ is any parameter sequence for $b$, then

$$
m_{n} \leq h_{n} \leq M_{n}, \quad n \geq 0 \text {. }
$$

We make one other observation concerning chain sequences for use in the next proof. Suppose a chain sequence $\left\{b_{n}\right\}$ determines its parameters uniquely, that is, its minimal and maximal parameters are equal. Then no other chain sequence can dominate it. In particular, if $\left\{d_{n}\right\}$ is also a chain sequence, an inequality of the form $d_{n}^{(1)} \geq b_{n} \quad(n \geq 1)$ is impossible since a chain sequence of the form $\left\{d_{n}^{(1)}\right\}$ cannot determine its parameters uniquely.

The next result does not require convergence of $\left\{\alpha_{n}\right\}$ for determinacy.

Theorem 4. Let $p>0$ and let

$$
\beta_{n}(p)=\frac{1}{4}-\frac{p(p-2)}{4(2 n+p-2)(2 n+p)}, \quad n \geq 1 .
$$

Then the Hmp is determined if $c_{n} \rightarrow \infty$ and

$$
\alpha_{n+1}(c) \leq \beta_{n}(p) \quad(n \geq 1) \quad \text { and } \quad \sum \frac{n^{p}}{c_{n}}=\infty .
$$

The Hmp is indeterminate if $\left\{\alpha_{n}(c)\right\}$ is a chain sequence and for some $p>1$, there exists a $k \geq 1$ such that

$$
\alpha_{n+k}(c) \geq \beta_{n}(p) \quad(n \geq 1) \quad \text { and } \quad \sum^{\prime} \frac{n^{p}}{c_{n}}<\infty .
$$

Proof. We have the identity

$$
\beta_{n}(p)=\left(1-\frac{n-1}{2 n+p-2}\right) \frac{n}{2 n+p} .
$$


It follows that $\left\{\beta_{n}(p)\right\}$ is a chain sequence with the (minimal) parameters $h_{n}=n /(2 n+p)$. Referring to the lemma, we see that the inequality (4.2) implies that the minimal parameters $m_{n}^{*}$ for $\left\{\alpha_{n}^{(1)}(c)\right\}$ satisfy $m_{n}^{*}(c) \leq h_{n}$. Thus we have $\left[1-m_{k}^{*}(c)\right] / m_{k}^{*}(c) \geq(k+p) / k$ and therefore, referring again to (3.4),

$$
A_{n} \geq \prod_{k=1}^{n-1} \frac{k+p}{k} \frac{2 n+p}{n\left(c_{n+2}-c\right)} \sim \frac{2 n^{p}}{c_{n+2}} .
$$

It then follows from Theorem 1 that (4.2) implies determinacy of the Hmp.

Turning to (4.3), we first explain the condition $p>1$. Set

$$
\pi_{n}(h)=\prod_{k=1}^{n} \frac{h_{k}}{1-h_{k}}=\prod_{k=1}^{n} \frac{k}{k+p} \sim n^{-p} .
$$

According to a criterion of Wall $[15 ; 8$, p. 101$],\left\{h_{n}\right\}$ is the maximal parameter sequence for $\left\{\beta_{n}(p)\right\}$ if and only if $\sum \pi_{n}(h)=\infty$. Therefore, if $0<p \leq 1$, the minimal parameters $h_{n}$ would also be maximal. According to the remark made immediately prior to this theorem, the first inequality in (4.3) would then be impossible if $\left\{\alpha_{n}(c)\right\}$ is a chain sequence.

We therefore take $p>1$ and suppose first that (4.3) is satisfied for $k=1$. The lemma implies that $m_{n}^{*}(c) \geq h_{n}$. Therefore we get (4.5) but with the inequality reversed so that $A_{n}$ is dominated by something that is asymptotically proportional to $n^{p} / c_{n+2}$. Thus, (4.3) implies that $\sum A_{n}$ converges and we conclude that the moment problem is indeterminate.

Next suppose (4.3) holds for $k=j+1, j \geq 1$. We then consider the "numerator polynomials of order $j$ " (the polynomials corresponding to the recurrence coefficients $c_{n}^{j}=c_{n+j}, \lambda_{n}^{j}=\lambda_{n+j}$ ) and conclude that the corresponding Hamburger moment problem is indeterminate. But Sherman [13] has noted that if the Hamburger moment problem for (1.1) is determined, so is the moment problem for the corresponding numerator polynomials (of (2.15)); hence by induction, the moment problem for the numerator polynomials of order $j$ is determined. It thus follows that the moment problem for (1.1) must be indeterminate.

Remark. If $-1<p \leq 0,\left\{\beta_{n}(p)\right\}$ remains a chain sequence and (4.2) would remain valid but the second condition would become simply $\sum^{\prime} c_{n}^{-1}=\infty$. However, as remarked following Theorem 3 , if $\left\{\alpha_{n}(c)\right\}$ is a chain sequence, the latter is sufficient for determinacy without further hypotheses.

Condition (4.2) with $p=1$ applies to Wilson's limiting case of the StieltjesCarlitz polynomials mentioned at the end of the previous section. Indeed, for this case, we actually have $\alpha_{n}=\beta_{n}(1)$ so Theorem 1 could be applied directly to the known parameters of $\left\{\beta_{n}(1)\right\}$ to conclude determinacy.

An example of $\left\{\alpha_{n}\right\}$ which converges to $\frac{1}{4}$ and for which neither (4.2) nor (4.3) can apply is the following. Let $\left\{n_{k}\right\}$ be a strictly increasing sequence of 
positive integers, $n_{1}>1$, such that $\sum n_{k}^{-1 / 2}<\infty$. Then choose $c_{n} \rightarrow \infty$ and $\lambda_{n+1}>0$ such that

$$
\alpha_{n}= \begin{cases}\frac{1}{4}\left(1-\frac{n+\sqrt{n-1}}{(n+1) \sqrt{n-1}}\right) & \text { if } n=1+n_{k}, \\ \frac{1}{4} & \text { if } n \neq n_{k}, 1+n_{k}, \\ \frac{1}{4}\left(1+\frac{n+\sqrt{n+1}}{n \sqrt{n}}\right) & \text { if } n=n_{k} .\end{cases}
$$

One can now verify directly that $\left\{\alpha_{n}\right\}$ is a chain sequence whose minimal parameters are $m_{n}$ given by $m_{n}=(\sqrt{n}+1) / \sqrt{4 n}$ if $n=n_{k}$ and $m_{n}=$ $n /(2 n+2)$ if $n \neq n_{k}$. Therefore using the notation of (4.6), we have for $n_{p} \leq N<n_{p+1}$

$$
\begin{aligned}
\alpha_{N}(m) & =\prod_{n=1}^{N} \frac{n}{n+2} \prod_{k=1}^{p} \frac{n_{k}+2}{n_{k}} \prod_{k=1}^{p} \frac{\sqrt{n_{k}}+1}{\sqrt{n_{k}}-1} \\
& =\frac{2}{(N+1)(N+2)} \prod_{k=1}^{p}\left(1+\frac{2}{n_{k}}\right) \prod_{k=1}^{p}\left(1+\frac{2}{\sqrt{n_{k}}-1}\right) .
\end{aligned}
$$

Since $\sum n_{k}^{-1 / 2}<\infty$, it follows that $\pi_{N}(m)$ is asymptotically proportional to $N^{-2}$, hence $\sum \pi_{n}(m)<\infty$ so the minimal parameters are not also maximal parameters. More pertinently, corresponding to (3.4), we here have that $A_{n}$ is asymptotically proportional to $n^{2} / c_{n}$. Thus if the recurrence coefficients produce $\alpha_{n}$ as in (4.7), the Hamburger moment problem is determined if and only if $\sum n^{2} / c_{n}=\infty$.

For the case $\alpha_{n}(c) \rightarrow L=0$, the determinacy criterion given by Theorem 3 , namely $\liminf _{n \rightarrow \infty} c_{n}^{1 / n}<\infty$, shows that it will require a rather prodigious growth rate by $c_{n}$ to yield an indeterminate moment problem. The only specific example we know of in which $L=0$ and $c_{n} \rightarrow \infty$ is the family of Charlier polynomials. Of course, for the latter, we have $c_{n}=O(n)$ and $\lambda_{n}=O(n)$ so determinacy is already given by Carleman's criterion. Since it seems unlikely that we will soon see explicit examples of orthogonal polynomials for which $L=0$ and $\lim _{n \rightarrow \infty} c_{n}^{1 / n}=\infty$, we will content ourselves here with giving a couple of examples which will illustrate how great must be the growth rate of $c_{n}$ to have indeterminate Hamburger moment problems. With the aid of comparison theorems (see the next section), these examples could be used to generate general classes of examples.

Our first example will have $\alpha_{n}=O\left(n^{-1}\right)$. Take $\alpha_{1} \leq 1 / 2$ and

$$
\alpha_{n+1}=\frac{n^{2}+1}{(n+1)^{2}(n+2)} .
$$

Then $\left\{\alpha_{n}\right\}$ is dominated by $\left\{(n+1)^{-1}\right\}$ and the latter is a chain sequence (with minimal parameters $n /(n+1)(n \geq 0))$. Thus if we take $c_{n}>0$, then the spectral interval will be contained in $[0, \infty)$. It is readily verified that the 
minimal parameters for $\left\{\alpha_{n}^{(1)}\right\}$ are $m_{n}^{*}=n /[(n+1)(n+2)]$. From these, we obtain correspondingly:

$$
A_{n}=\frac{\left(1+2^{2}\right) \cdots\left(1+n^{2}\right)(n+1)(n+2)}{n ! c_{n+2}}=\frac{(n+2) !}{c_{n+2}} \prod_{k=1}^{n}\left(1+\frac{1}{k^{2}}\right) .
$$

It follows that the corresponding Hamburger moment problem is determined if and only if $\sum n ! c_{n}^{-1}=\infty$.

For a second example with more rapid convergence of $\left\{\alpha_{n}\right\}$ to 0 , take $c_{n}>0$, $\alpha_{1}=q(0<q<1)$ and

$$
\alpha_{n+1}=\left(1-q^{n}\right) q^{n+1}, \quad n \geq 1 .
$$

Then $\left\{\alpha_{n}\right\}$ is a chain sequence so again the spectral interval is contained in $[0, \infty)$. The sequence $\left\{q^{n}\right\}$ is a nonminimal parameter sequence for $\left\{\alpha_{n}\right\}$ so for the corresponding minimal parameters we have this time: $m_{n}^{*}<q^{n+1}$ hence

$$
A_{n}>\frac{(q ; q)_{n}}{q^{n(n+3) / 2} c_{n+2}}
$$

where, as usual, $(q ; q)_{n}=(1-q)\left(1-q^{2}\right) \cdots\left(1-q^{n}\right)$. It follows that the moment problem will be determined even if $c_{n}^{-1}=O\left(q^{n(n-1) / 2}\right)$.

\section{COMPARISON THEOREMS AND OTHER CRITERIA}

The argument based on the lemma that was used to prove Theorem 4 can be used to obtain in a slightly more general form a comparison theorem previously obtained in [6]. Application of this comparison theorem to the last two examples of the preceding section would then generate classes of examples involving recurrence coefficients for which $\alpha_{n} \rightarrow 0$.

Let $F=\left\{F_{n}(x)\right\}$ be a second $O P S$ given by the recurrence formula

$$
\begin{aligned}
& F_{n}(x)=\left(x-f_{n}\right) F_{n-1}(x)-\rho_{n} F_{n-2}(x), \\
& F_{-1}(x)=0, \quad F_{0}(x)=1 .
\end{aligned}
$$

Suppose that there exists a real $f$ such that $f_{n}>f(n \geq 1)$ and

$$
\delta_{n}(f)=\frac{\rho_{n+1}}{\left(f_{n}-f\right)\left(f_{n+1}-f\right)} \leq \frac{\lambda_{n+1}}{\left(c_{n}-c\right)\left(c_{n+1}-c\right)} .
$$

Then if (2.2) is satisfied, $\left\{\delta_{n}(f)\right\}$ is a chain sequence and the chain sequence $\left\{\delta_{n}^{(1)}(f)\right\}$ has minimal parameters $p_{n}^{*}$ that satisfy $p_{n}^{*} \leq m_{n}^{*}(n \geq 0)$ (by the lemma). It follows that (referring once more to (3.4))

$$
A_{n}(F) \geq A_{n}(P) \cdot \frac{c_{n+2}-c}{f_{n+2}-f} .
$$

This yields the following theorem. 
Theorem 5. Let (2.2) be satisfied and let the associated Hamburger moment problem be determined. If (5.2) is satisfied and if $f_{n}=O\left(c_{n}\right)$, then the Hamburger moment problem for (5.1) is also determined.

Two comparison theorems that apply without the hypothesis that the spectral interval is bounded on one side were obtained by Carleman [4, pp. 214-215]. These were given in [14, pp. 59-60] but the second is mistated there. Using the notation of this paper, Carleman's criteria can be stated as follows.

Assume that either

(i) the sequences $\left\{f_{n}-c_{n}\right\}$ and $\left\{\rho_{n}^{1 / 2}-\lambda_{n}^{1 / 2}\right\}$ are bounded; or

(ii) there exist positive constants $A, B$ and $k_{n}$ such that $A \leq k_{n} \leq B$ and

$$
f_{n}=k_{n} c_{n}, \quad \rho_{n+1}=k_{n} k_{n+1} \lambda_{n+1}, \quad n \geq 1 \text {. }
$$

Then if the Hamburger moment problem for (1.1) is determined (without assuming condition (2.2)), so is the Hamburger moment problem for (5.1) determined. (In [14], the second part of (5.4) is misprinted as $\rho_{n+1}=\lambda_{n+1}$.)

For the symmetric case $\left(c_{n}=0\right.$ for all $\left.n\right)$, the question of determinacy of the Hamburger moment problem can be completely described. In fact, if we consider the symmetric recurrence relation in the form (2.10), there are 4 additional moment problems associated with (2.10). These are the Hamburger and Stieltjes moment problems corresponding to the orthogonal polynomials $P_{n}(x)$ and with the corresponding kernel polynomials $K_{n}(x)$ whose symmetrizations lead to the orthogonal polynomials $S_{n}(x)$ satisfying (2.10). If we define

$$
G_{n}=\frac{\gamma_{2} \gamma_{4} \cdots \gamma_{2 n}}{\gamma_{3} \gamma_{5} \cdots \gamma_{2 n+1}}, \quad H_{n}=\frac{\gamma_{3} \gamma_{5} \cdots \gamma_{2 n+1}}{\gamma_{4} \gamma_{6} \cdots \gamma_{2 n+2}}
$$

the determinacy-indeterminacy for all 5 moment problems can be decided on the basis of the convergence-divergence of the two series $\sum G_{n}$ and $\sum H_{n}$ (see [10]). In particular, the Hamburger moment problem for the symmetric polynomials is determined if and only if at least one of the two series above diverges. The application of Carleman's comparison theorems (after perhaps first performing a translation in (2.10) for application of $(5.4)$ ) then will yield determinacy criteria for some limited classes of nonsymmetric recurrence relations in which the spectrum reaches from $-\infty$ to $+\infty$. These will be recurrences of the form (1.1) with $\left\{c_{n}\right\}$ bounded however.

We remark that we find it interesting that in the second of Carleman's criteria, we have $\rho_{n+1} /\left(f_{n} f_{n+1}\right)=\lambda_{n+1} /\left(c_{n} c_{n+1}\right)$. The same ratio that is so prominent in our analysis of the case where the spectrum is bounded on one side may play some sort of role in the general case also.

We note here that a second criterion for determinacy that applies when the spectrum is not restricted to a half line is the following due to Dennis and Wall [12] (see also [1, p. 25]). Let $\theta_{n}=\left|c_{n}\right|\left(\lambda_{n} \lambda_{n+1}\right)^{-1 / 2}$. The Hamburger moment problem is determined if the series $\sum \theta_{n}$ diverges. Unlike Carleman's criterion (1.6), the Dennis and Wall criterion involves both coefficient sequences from the recurrence relation. However, in the case, $c_{n} \rightarrow \infty, \lambda_{n} \rightarrow L$ (especially 
with $L=\frac{1}{4}$ which we believe is a very significant case), both criteria are "similar" in that they would require divergence of $\sum D_{n}$ with $D_{n}=\left(c_{n} c_{n+1}\right)^{-1 / 2}$ (Carleman) or $D_{n}=\left(c_{n-1} c_{n+1}\right)^{-1 / 2}$ (Dennis and Wall).

Two interesting criteria for determinacy which, at first glance, appear quite general are due to Wouk [18, p. 147]. The first criterion is that the moment problem is determined if one of the following holds for $n \rightarrow \infty$ :

$$
\text { (i) } \lambda_{n}^{1 / 2}+c_{n}+\lambda_{n+1}^{1 / 2}=0(1), \quad \text { (ii) } \lambda_{n}^{1 / 2}-c_{n}+\lambda_{n+1}^{1 / 2}=0(1) \text {. }
$$

The first of these can be transformed into the second by a reflection: $x \rightarrow-x$ so suppose (ii) holds. Then there exists an $M$ such that

$$
\begin{aligned}
c_{n}+M & >\sqrt{\lambda_{n}}+\sqrt{\lambda_{n+1}}, \\
\alpha_{n}(-M)=\frac{\lambda_{n+1}}{\left(c_{n}+M\right)\left(c_{n+1}+M\right)} & <\frac{\lambda_{n+1}}{\left(\sqrt{\lambda_{n}}+\sqrt{\lambda_{n+1}}\right)\left(\sqrt{\lambda_{n+1}}+\sqrt{\lambda_{n+2}}\right)} \\
& =\left(1-\frac{\sqrt{\lambda_{n}}}{\sqrt{\lambda_{n}}+\sqrt{\lambda_{n+1}}}\right) \frac{\sqrt{\lambda_{n+1}}}{\sqrt{\lambda_{n+1}}+\sqrt{\lambda_{n+2}}} .
\end{aligned}
$$

That is, $c_{n}>-M$ and $\left\{\alpha_{n}(-M)\right\}$ is a chain sequence, hence the spectrum is contained in $[-M, \infty)$. Thus, the criteria only apply to those cases where the spectra are bounded on one side.

The second criterion given by Wouk is that the Hamburger moment problem is determined if $\sum \lambda_{n}^{-1 / 4}=\infty$ and if the related Jacobi matrix is positive. Wall and Wetzel's quadratic form characterization of chain sequences [16] shows that positivity of the Jacobi matrix implies that again, $\left\{\alpha_{n}(-M)\right\}$ is a chain sequence (after a reflection) so that once again the spectrum is contained in a half-line. Wouk obtains these criteria by Hilbert space methods but Akhiezer [1, pp. 25-27] shows how they can be obtained directly from the recurrence relation (1.1).

A criterion that implies indeterminacy due to Brezeshinsky is also derived in [1, p. 28]. This result says that the moment problem is indeterminate if $\left|c_{n}\right|<M \quad(n \geq 1)$, and

$$
\sum \frac{1}{\lambda_{n}}=\infty \text { and } \lambda_{n-1} \lambda_{n+1} \leq \lambda_{n}^{2} \text { for all sufficiently large } n \text {. }
$$

Let us first consider the symmetric case $c_{n}=0$. Writing $\gamma_{n}=\lambda_{n}$ and referring to (5.5), we see that the second inequality in (5.6) shows that there are positive constants $G$ and $H$ such that $G_{n}<G \gamma_{2 n+1}^{-1}$ and $H_{n}<H \gamma_{2 n+2}^{-1}$. Therefore, by the criteria for the symmetric case, convergence of $\sum \lambda_{n}^{-1}$ implies that the Hamburger moment problem is indeterminate. Application of the first of Carleman's comparison theorems then shows the moment problem is indeterminate in the general case. 


\section{Concluding EXAMPles}

We have noted a number of specific examples to which our results apply. As an indicator of directions in which further research is needed, we conclude with an important family of orthogonal polynomials for which, in many cases, our methods (and all others that we are aware of) fail to yield conclusions.

Askey and Ismail have recently studied intensively several classes of orthogonal polynomials [3]. This important work analyzes, among others, the orthogonal polynomials whose monic form satisfies (1.1) with

$$
c_{n+1}=a q^{-n}, \quad \lambda_{n+1}=\left(1-q^{n}\right)\left(b-c q^{n-1}\right) q^{1-2 n}, \quad 0<|q|<1,
$$

where either

(i) $0<q<1, b \geq 0$ and $b>c$, or

(ii) $-1<q<0, b<0, b<c<b / p$.

By explicitly determining the asymptotic behavior of the terms in the two orthonormal series of (2.19), Askey and Ismail deduce the determinacy or indeterminacy of the associated Hamburger moment problems for all cases. Additionally, for those cases where the moment problem is determined, they explicitly obtain the precise orthogonality relations. They show that the associated Hamburger moment problem is determined if and only if

$$
a^{2}>4 b \text { and }|q| \geq \frac{|a|-\sqrt{a^{2}-4 b}}{|a|+\sqrt{a^{2}-4 b}} .
$$

Considering criteria based on recurrence coefficients, we first observe that if $a=0$, then the criterion referred to following (5.5) shows that the Hamburger moment problem is always indeterminate. If $a \neq 0$, there is no loss of generality in assuming $a>0$. Then in case (ii) $(q<0)$ we see that $\inf c_{n}=-\sup c_{n}=-\infty$ so the spectrum extends from $-\infty$ to $+\infty$ and our methods fail completely. None of the other criteria that we know of provide any information about this case either.

When $q>0$, we have $c_{n} \rightarrow \infty$ and $\lambda_{n} \rightarrow L=b / a^{2}\left(2 a \mu=a-\sqrt{a^{2}-4 b}\right)$. Therefore, when $a^{2}>4 b$, Theorem 2 says the moment problem is determined if $q>\mu /(1-\mu)$ and indeterminate if $q<\mu /(1-\mu)$. These results are consistent with conclusions arrived at by Askey and Ismail but leave open the case when $q=\mu /(1-\mu)$. For the latter case, if we also have $b+c q^{-1}>$ 0 , then $\left\{\alpha_{n}\right\}$ is nondecreasing and Theorem 3 yields the conclusion that the moment problem is determined but our methods leave the remaining case open. Under the same conditions, Wouk's first-cited theorem also applies and leads to the same conclusion. Askey and Ismail's work shows the moment problem is determined in all cases if $q=\mu /(1-\mu)$.

If $a^{2}=4 b$, then for $N$ sufficiently large, $\alpha_{n+N}<\beta_{n}(1)$ (where $\beta_{n}(p)$ is still given by (5.4)). Thus $\left\{\alpha_{n}\right\}$ is an eventual chain sequence and (2.2) is satisfied. Theorem 4 now shows the moment problem is indeterminate (take $p>2$ in (4.3)). 
Finally, if $a^{2}<4 b$, then $\left\{\alpha_{n}(c)\right\}$ cannot be a chain sequence for any $c$ and the spectrum extends from $-\infty$ to $+\infty$ again. None of the recurrence based criteria appear to apply to this case although Askey and Ismail have shown that the Hamburger moment problem is indeterminate. Thus there are large classes of recurrence coefficients to which none of the known criteria come close to handling. Much work remains to be done.

\section{REFERENCES}

1. N. I. Ahiezer, The classical moment problem, Hafner, New York, 1965.

2. W. A. Al-Salam and L. Carlitz, Some orthogonal q-polynomials, Math. Nachr. 30 (1965), 4761.

3. R. Askey and M. E. H. Ismail, Recurrence relations, continued fractions and orthogonal polynomials, Mem. Amer. Math. Soc., no. 300, 1984.

4. T. Carleman, Sur les équations intégrales singulières à noyau réel et symmétrique, Uppsala Universitets Arsskrift, 1923, 228 pp.

5. T. S. Chihara, Chain sequences and orthogonal polynomials, Trans. Amer. Math. Soc. 104 (1962), 1-16.

6 . $\ldots$, On recursively defined orthogonal polynomials, Proc. Amer. Math. Soc. 16 (1965), 702710.

7. $\_$, Convergent sequences of orthogonal polynomials, J. Math. Anal. Appl. 38 (1972), 335347.

8. __ An introduction to orthogonal polynomials, Gordon and Breach, New York, 1978.

9. __ Orthogonal polynomials whose distributions have finite point spectra, SIAM J. Math. Anal. 11 (1980), 358-364.

10. __ Indeterminate symmetric moment problems, J. Math. Anal. Appl. 85 (1982), 331-346.

11. __ Spectral properties of orthogonal polynomials on unbounded sets, Trans. Amer. Math. Soc. 270 (1982), 623-639.

12. J. J. Dennis and H. S. Wall, The limit circle case for a positive-definite J-fraction, Duke Math. J. 12 (1945), 255-273.

13. J. Sherman, On the numerators of the convergents of the Stieltjes continued fractions, Trans. Amer. Math. Soc. 35 (1933), 64-87.

14. J. Shohat and J. D. Tamarkin, The problem of moments, Math. Surveys, no. 1, Amer. Math. Soc., Providence, R.I., 1943/1950.

15. H. S. Wall, The analytic theory of continued fractions, Van Nostrand, New York, 1948.

16. H. S. Wall and Marion Wetzel, Contributions to the analytic theory of continued fractions, Trans. Amer. Math. Soc. 55 (1944), 373-397.

17. J. Wilson, Some hypergeometric orthogonal polynomials, SIAM J. Math. Anal. 10 (1980), 690701.

18. A. Wouk, Difference equations and J-matrices, Duke Math. J. 20 (1953), 141-159.

Department of Mathematical Sciences, Purdue University Calumet, Hammond, IndiANA 46323 